\title{
Pregabalin and Libido- Case Reports
}

\author{
Mihaela Bucur* and Pablo Jeczmien
}

Linwood CMHS, Sussex Partnership NHS Trust, Butlers Green Road, Haywards Heath, West Sussex, United Kingdom, Brighton and Sussex Medical School, United Kingdom

\begin{abstract}
Pregabalin is a drug used in UK for the following conditions: peripheral and central neuropathic pain, adjunctive therapy for partial seizures with or without secondary generalization and generalized anxiety disorder. Drugs used to treat anxiety disorders very often do have sexual side effects (e.g. SSRIs, SNRIs, and Benzodiazepines). Patients with generalized anxiety disorder can have an impaired libido due to the direct effect of anxiety.

Since mid 2009, when we first started prescribing Pregabalin for patients with a diagnosis of Generalized Anxiety Disorder, two male patients reported enhanced libido after having started treatment with Pregabalin. It would be argued that the Pregabalin mechanism of action might be responsible for these changes. An extensive search of the medical literature conducted in 2010 failed to retrieve any relevant published studies or case reports that comment on the impact of Pregabalin on increased libido.
\end{abstract}

Keywords: Anxiety, pregabalin, libido.

\section{PREGABALIN AND LIBIDO}

Pregabalin is a drug used in the treatment of neuropathic pain, partial seizures and generalized anxiety disorder. Pregabalin inhibits the release of the excess excitatory neurotransmitters, presumably by binding to the alpha 2 delta proteins of the widely distributed voltage dependent calcium channels in spine and brain. This mechanism is responsible for the anxyolitic, anticonvulsant and antinocioceptive effect. [1] Libido is the term used to describe sexual desire or the psychic and emotional energy related to sexuality. In the accepted sense, libido refers specifically to the mental manifestations of the sexual instinct. [2] Drugs used to treat anxiety disorders very often do have sexual side effects. Patients with generalized anxiety disorder can have an impaired libido due to the direct effect of anxiety.

Since 2009, when we have started prescribing Pregabalin for community patients with Generalized Anxiety Disorder, two patients self reported enhanced libido shortly after having started treatment with Pregabalin.

The first case is a 42-year-old gentleman who has a diagnosis of Alcohol Dependence, Narcissistic Personality Disorder associated with Generalized Anxiety Disorder. His only pharmacological treatment was Pregabalin $150 \mathrm{mg}$ (twice daily) and Quetiapine 50mg (twice daily). Pregabalin was introduced three weeks before he reported increased libido. He was in a stable relationship with a lady he met four years ago. Although he was off work for about 6 months, no significant life events were identified since he started Pregabalin. The above pharmacological treatment was the mainstay of his management plan when he selfreported increased libido.

*Address correspondence to this author at the Linwood CMHS, Sussex Partnership NHS Trust, Butlers Green Road, Haywards Heath, West Sussex, United Kingdom; Tel: 00441444416606; Fax: 00441444413884;

E-mail:msbukuk@yahoo.co.uk
The second case, a 52-year-old gentleman who had a diagnosis of Major Depression and Generalized Anxiety Disorder, self reported increased libido approximately four weeks after he started Pregabalin. He started to take Pregabalin approximately four months after his discharge from an in-patient ward where he has been admitted to manage his suicide risk associated with severe depression. Additional prescribed medication was Venlafaxine 150 $\mathrm{mg} /$ day which the patient started to take four months before he reported increased libido. He was in a supportive relationship that started about eleven years ago and he denied any associated social circumstances that may have contributed to the increased libido.

Both patients did not have other significant medical history.

The above-presented cases may suggest a temporal association between starting treatment with Pregabalin and the increased libido. Pregabalin has a mechanism of action that it is different from the other psychotropic medications indicated in the treatment of Generalized Anxiety Disorder like: benzodiazepines, azapirones, selective serotonin reuptake inhibitors or serotonin noradrenalin reuptake inhibitors [3]. Libido is related to different factors like: hormones levels, the psychosocial context and it is considered to be increased by dopamine and decreased by prolactine. In the book Essential Psychopharmacology, Stahl discussed about libido as being hypothetically a dopaminergic phenomenon mediated by the mesolimbic dopaminergic "reward centre". Prolactin is hypothesized to have a negative influence on sexual desire, but its action is relatively poorly understood. [4]

Pregabalin is inactive at serotonin and dopamine receptors, it does not inhibit dopamine, serotonin or noradrenalin uptake hence a possible explanation for its impact on libido it cannot be made without in depth research. 
We hope that our case reports can be an incentive for initiating a thorough research to analyse the association between Pregabalin and libido and the overall impact on compliance and quality of life.

\section{REFERENCES}

[1] Rickels K, Pollack MH, Feltner DE, et al. Pregabalin for treatment of generalized anxiety disorder: 14week, multicenter, double blind,

Received: March 03, 2011
Placebo-Controlled Trial of Pregabalin and Alprazolam. Arch Gen Psychiatry 2005; 62: 1022-30.

[2] Sadock BJ, Sadock VA. Kaplan and Sadock's synopsys of psychiatry: Behavioural Sciences Clinical Psychiatry. Tenth, North American ed. Baltimore: Lippincott Williams and Wilkins 2007.

[3] Pohl R, Feltner D, Fieve R, Pande AC. An efficacy of pregabalin in the treatment of generalized anxiety disorder. J Clin Psychopharmacol 2005; 25: 151-58.

[4] Stahl S. Essential Psychopharmacology, chapter 14 Sex-specific and sexual function-related psychopharmacology. USA: Cambridge University Press 2000.

(C) Bucur and Jeczmien; Licensee Bentham Open.

This is an open access article licensed under the terms of the Creative Commons Attribution Non-Commercial License (http://creativecommons.org/licenses/by-nc/3.0/) which permits unrestricted, non-commercial use, distribution and reproduction in any medium, provided the work is properly cited. 